\title{
Front Matter: Volume 10960
}

, "Front Matter: Volume 10960," Proc. SPIE 10960, Advances in Patterning Materials and Processes XXXVI, 1096001 (23 May 2019); doi:

$10.1117 / 12.2532428$

SPIE. Event: SPIE Advanced Lithography, 2019, San Jose, California, United States 


\title{
PROCEEDINGS OF SPIE
}

\section{Advances in Patterning Materials and Processes XXXVI}

\author{
Roel Gronheid \\ Daniel P. Sanders \\ Editors
}

25-28 February 2019

San Jose, California, United States

Sponsored and Published by

SPIE 
The papers in this volume were part of the technical conference cited on the cover and title page. Papers were selected and subject to review by the editors and conference program committee. Some conference presentations may not be available for publication. Additional papers and presentation recordings may be available online in the SPIE Digital Library at SPIEDigitallibrary.org.

The papers reflect the work and thoughts of the authors and are published herein as submitted. The publisher is not responsible for the validity of the information or for any outcomes resulting from reliance thereon.

Please use the following format to cite material from these proceedings:

Author(s), "Title of Paper," in Advances in Patterning Materials and Processes XXXVI, edited by Roel Gronheid, Daniel P. Sanders, Proceedings of SPIE Vol. 10960 (SPIE, Bellingham, WA, 2019) Sevendigit Article CID Number.

ISSN: 0277-786X

ISSN: 1996-756X (electronic)

ISBN: 9781510625679

ISBN: 9781510625686 (electronic)

Published by

SPIE

P.O. Box 10, Bellingham, Washington 98227-0010 USA

Telephone +1 3606763290 (Pacific Time) · Fax +1 3606471445

SPIE.org

Copyright @ 2019, Society of Photo-Optical Instrumentation Engineers.

Copying of material in this book for internal or personal use, or for the internal or personal use of specific clients, beyond the fair use provisions granted by the U.S. Copyright Law is authorized by SPIE subject to payment of copying fees. The Transactional Reporting Service base fee for this volume is $\$ 18.00$ per article (or portion thereof), which should be paid directly to the Copyright Clearance Center (CCC), 222 Rosewood Drive, Danvers, MA 01923. Payment may also be made electronically through CCC Online at copyright.com. Other copying for republication, resale, advertising or promotion, or any form of systematic or multiple reproduction of any material in this book is prohibited except with permission in writing from the publisher. The CCC fee code is $0277-$ $786 \times / 19 / \$ 18.00$.

Printed in the United States of America.

Publication of record for individual papers is online in the SPIE Digital Library.

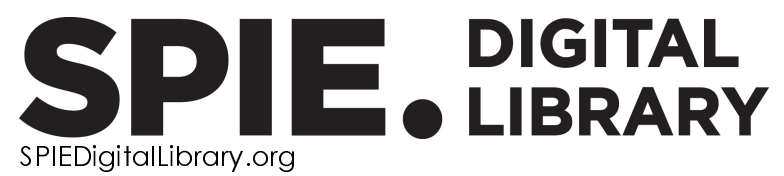

Paper Numbering: Proceedings of SPIE follow an e-First publication model. A unique citation identifier (CID) number is assigned to each article at the time of publication. Utilization of CIDs allows articles to be fully citable as soon as they are published online, and connects the same identifier to all online and print versions of the publication. SPIE uses a seven-digit CID article numbering system structured as follows:

- The first five digits correspond to the SPIE volume number.

- The last two digits indicate publication order within the volume using a Base 36 numbering system employing both numerals and letters. These two-number sets start with $00,01,02,03,04$, 05, 06, 07, 08, 09, OA, OB ... 0Z, followed by 10-1Z, 20-2Z, etc. The CID Number appears on each page of the manuscript. 


\title{
Contents
}

\author{
ix Authors \\ xii Conference Committee
}

STOCHASTICS AND EXPOSURE MECHANISMS: JOINT SESSION WITH CONFERENCES 10960 AND 10957

1096008 Multiscale approach for modeling EUV patterning of chemically amplified resist [10960-7]

1096009 The hidden energy tail of low energy electrons in EUV lithography [10960-8]

EUV RESISTS

10960 OA PSCAR optimization to reduce EUV resist roughness with sensitization using Resist Formulation Optimizer (RFO) [10960-9]

10960 OB Advanced EUV negative tone resist and underlayer approaches exhibiting sub-20nm half-pitch resolution [10960-10]

10960 OC Multi-trigger resist: novel synthesis improvements for high resolution EUV lithography [10960-1 1]

10960 OD Improvement of dual insolubilization resist performance through the incorporation of various functional units [10960-12]

\section{RESIST FUNDAMENTALS}

$109600 \mathrm{G}$ Understanding the photoacid generator distribution at nanoscale using massive cluster secondary ion mass spectrometry [10960-15]

10960 ol Roughness power spectral density as a function of aerial image and basic process/resist parameter [10960-17]

\section{INTEGRATION}

10960 OK Addressing challenges in the mitigation of stochastic effects [10960-19] 
$10960 \mathrm{OL} \quad$ Exploration of EUV-based self-aligned multipatterning options targeting pitches below $20 \mathrm{~nm}$ [10960-20]

10960 ON Self-aligned fin cut last patterning scheme for fin arrays of 24nm pitch and beyond [10960-22]

MONOLAYER MATERIALS IN DEVICE FABRICATION

$109600 Q \quad$ Selective spin-on deposition of polymers on heterogeneous surfaces [10960-25]

10960 OR Ultra-thin conformal coating for spin-on doping applications [10960-26]

DIRECTED SELF-ASSEMBLY I: JOINT SESSION WITH CONFERENCES 10960 AND 10958

10960 OU Post-polymerization modification of PS-b-PMMA for achieving directed self-assembly with sub10nm feature size [10960-50]

DIRECTED SELF-ASSEMBLY II: DEFECTIVITY

10960 OV Kinetics of defect annihilation in chemo-epitaxy directed self-assembly [10960-30]

10960 0W Pattern defect reduction for chemo-epitaxy DSA process [10960-31]

10960 OY Defect mitigation in sub-20nm patterning with high-chi, silicon-containing block copolymers [10960-33]

$109600 Z$ Accelerate the analysis and optimization of lamellar BCP process using machine learning [10960-34]

STUDENT SESSION

1096010 ToF-SIMS analysis of antimony carboxylate EUV photoresists [10960-35]

$1096011 \quad$ Modeling of novel resist technologies [10960-36]

1096012 Imaging behavior of highly fluorinated molecular resists under extreme UV radiation [10960-37]

iv 
MATERIAL SUPPLIER

$1096013 \quad$ Evolution of lithographic materials enabling the semiconductor industry [10960-38]

1096015 Development of metal organic cluster EUV photoresists [10960-40]

1096017 Expanding the lithographer's toolkit to reduce variability: filtration considerations [10960-42]

1096018 Start-up performance and pattern defectivity improvement using 2nm rated nylon filter developed with lithography filtration expertise [10960-43]

\section{UNDERLAYERS}

1096019 High temperature spin on carbon materials with excellent planarization and CVD compatibility [10960-44]

$109601 \mathrm{~A} \quad$ Improved hemicellulose spin on carbon hardmask [10960-45]

10960 1B Towards pure carbon: ultra-high carbon fullerene based spin-on organic hardmasks [10960-46]

10960 1D Development of novel thick spin-on carbon hardmask [10960-48]

POSTER SESSION: DSA

$109601 G \quad$ Micro-phase separation behavior study of the same system of a novel block copolymer (PS-b-PC) [10960-53]

$1096011 \quad$ Influence of PDI and composition ratio for micro phase separation about PS-b-PMMA block copolymer [10960-55]

$10960 \mathrm{lJ} \quad$ Phase behavior of polymer blend materials for polystyrene-b-polycarbonate (PS-b-PC) block copolymers and corresponding homopolymer polystyrene [10960-56]

$109601 \mathrm{~L} \quad$ Block copolymer line roughness and annealing kinetics as a function of chain stiffness [10960-80]

$109601 \mathrm{M} \quad$ Mitigation of line edge roughness and line width roughness in block copolymer directed selfassembly through polymer composition molecular weight manipulation [10960-81] 
POSTER SESSION: EUV

$109601 \mathrm{~N} \quad$ Defect conscious approaches in EUV patterning [10960-58]

10960 IP Robustness of interactive pattern fidelity error as a quality metric for integrated patterning [10960-60]

$109601 Q \quad$ Oligomers of MORE: Molecular Organometallic Resists for EUV [10960-61]

10960 IR Radical sensitive zinc-based nanoparticle EUV photoresists [10960-62]

\section{POSTER SESSION: FILTRATION}

$109601 \mathrm{U} \quad$ An exploration of the use of fluoropolymers in photofiltration [10960-66]

$109601 \mathrm{~V} \quad$ Bridging the defect gap in EUV photoresist [10960-67]

$109601 \mathrm{lX} \quad$ Filter technology developments to address defectivity in leading-edge photoresists [10960-69]

$109601 Y \quad$ A new tailored point-of-use filter to reduce immersion lithography downtime and defects [10960-70]

POSTER SESSION: FUNDAMENTALS

$1096021 \quad$ Study of outgassing from the ArF CA chemically amplified resist ArF (193 nm) exposure [10960-73]

1096022 Contact hole shrink of 193nm NTD immersion resist [10960-74]

\section{POSTER SESSION: UNDERLAYER}

$1096023 \quad$ Ordered polymer-based spin-on dopants [10960-75]

1096024 Development of new maleimides applied to spin-on carbon hardmask with characteristics of high heat resistance and good planarization [10960-76]

$1096025 \quad$ New silicon hard mask material development for sub-5nm node [10960-77] 
Application of downstream plasma generated radical methylation treatment to passive amorphous Si surface from TMAH etching during lithography process [10960-78]

$1096027 \quad$ Charge dissipation by use of a novel aqueous based quaternary ammonium compound for use in electron beam lithography on non-conductive substrates [10960-79] 
Proc. of SPIE Vol. $109601096001-8$

Downloaded From: https://www.spiedigitallibrary.org/conference-proceedings-of-spie on 26 Apr 2023 Terms of Use: https://www.spiedigitallibrary.org/terms-of-use 


\section{Authors}

Numbers in the index correspond to the last two digits of the seven-digit citation identifier (CID) article numbering system used in Proceedings of SPIE. The first five digits reflect the volume number. Base 36 numbering is employed for the last two digits and indicates the order of articles within the volume. Numbers start with 00, 01, 02, 03, 04, 05, 06, 07, 08, 09, OA, OB...0Z, followed by 10-12, 20-2Z, etc.

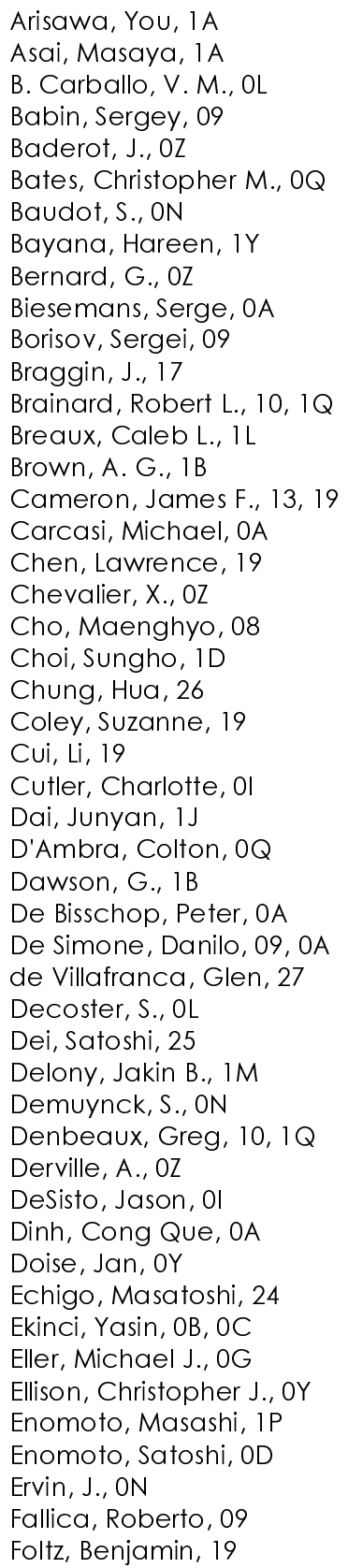


Kitano, Takahiro, OW Kobayashi, Shinji, IP Koh, Jai Hyun, OY Kohyama, T., 1V, 1X Kondo, Yoshihiro, OA Kosaka, Terumasa, 11 Kosma, Vasiliki, 1R Kozawa, Takahiro, OD Kwok, Amy, 22 LaBeaume, Paul, 19 Lada, T., 1B Laukkanen, Markus, OB Lazzarino, F., OL Lee, Byunghoon, 08 Lee, Choong Bong, 01 Lee, Hyungwoo, 08 Lee, Jin-Kyun, 12 Lee, Sangsul, 12

Li, Haochen, 26

Li, Jiajing, OV

Li, Mingqi, 0G, OR, 22, 23

Lim, Sanghak, 1D

Limary, Ratchana, OR, 23

Liu, Cong, 22

Liu, Weichen, 1G, $1 \mathrm{~J}$

Long, Luke, 11

Lopez, Gerald, 27

Lorant, C., OL

Lorusso, Gian, 09

Lu, Xinliang, 26

Ludovice, Peter J., 1L, 1M Luong, Nguyen Dang, OB Ma, Shawming, 26

Machida, Kohei, OD

Mack, Chris, Ol

Makinoshima, Takashi, 24

Mamiya, Hiroaki, OU

Mannaert, Geert, OV, OY

Mao, Guoping, 1J

Martinez, S., $\mathrm{OZ}$

Matsuki, Ryota, 11

Matsumoto, Yoko, 21

McClelland, A. L., OC, 1B

Meliorisz, Balint, OA

Meng, Lingkuan, $1 \mathrm{G}$

Miki, Yasushi, 24

Milenin, A. P., ON

Minami, Hiroko, 21

Minekawa, Yukie, OA

Miura, K., $1 \mathrm{X}$

Miyagi, Ken, OU

Mizuno, Takehito, 18

Montgomery, W., 1B

Moon, Junghwan, 08

Morikita, Shinya, $1 \mathrm{P}$

Morita, Kazuyo, 1A

Motono, Tomohiro, $1 \mathrm{~A}$

Mun, Jeong-Seok, 12

Murakami, Tetsuya, 18
Muramatsu, Makoto, oW

Murphy, Michael, 10, 1Q

Nafus, Kathleen, OA

Nagahara, Seiji, OA

Nakagawa, Hisashi, 18

Nakashima, Hideo, OA

Nakayama, Chisayo, 1A

Nannarone, Stefano, 09

Naulleau, Patrick P., 11

Nealey, Paul F., OV

Nelson, John, $\mathrm{Ol}$

Neureuther, Andrew R., 11

Ngunjiri, Johnpeter, 19

Nishi, Takanori, OW

Nishino, Tomoki, 21

Novak, Steven, 10

Ober, Christopher K., 15, 1R

O'Callaghan, G., OC

Ogaki, Ryosuke, 11

Oh, Hyuntaek, 12

Okada, Soichiro, 1P

Pan, Wenyang, 15

Park, James, 22

Park, Jong, 22

Park, Soyeon, 1D

Park, Sungwoo, 08

Petersen, John S., OA

Pieczulewski, Charles, $1 \mathrm{~A}$

Popere, Bhooshan C., OR, 19, 23

Popescu, C., OC

Ran, Ruicheng, $1 \mathrm{~J}$

Rantala, Juha, OB

Rena, Rochelle, 0 I

Rezvani, Seyed Javid, 09

Rincon-Delgadillo, Paulina, OV, OY

Robinson, Alex P. G., OC, 1B

Roth, J., OC

Sakai, Kazunori, 15, 1R

Sakai, Tatsuya, 25

Sato, Takashi, 24

Satoh, Toshifumi, OU

Schweikert, Emile A., OG

Segalman, Rachel A., OQ, OR, 23

Sekiguchi, Atsushi, 21

Seko, Tomoaki, 25

Serizawa, Ryuuichi, 25

Shao, Grant, 27

Shimada, Ryo, OA

Shimura, Satoru, 1P

Shin, Seungwook, 1D

Shiraishi, Gosuke, OA

Shiu, Eric, 18

Soussou, A., ON

Stock, Hans-Jürgen, OA

Stokes, Harold, $1 \mathrm{~A}$

Suh, Hyo Seon, OV, OY

Sung, Jin Wuk, 22

Takei, Kiniharu, 23

Takeshita, Kazuhiro, OA 
Tamaddon, A.-H., OL

Tanaka, Hiroki, 1 A

Tanaka, Yasuaki, 1A

Tanaka, Yuji, 1A

Thackeray, James W., 0l, 13

Theis, W., OC

Thompson, Andrew, 27

Tomono, Masaru, OA

Trefonas, Peter, OG, OI, OR, 23

Tsuzuki, Shuichi, 18

Ueno, Masayoshi, 24

Umeda, Toru, 18

Vandenberghe, Geert, 09, 0A, OY

Vangoidsenhoven, D., OL

Verkhoturov, Stanislav V., OG

Vincent, B., ON

Wang, S., ON

Weckx, P., ON

Wei, Yayi, $1 \mathrm{~J}$

Weires, Maximilian, $1 Q$

Willson, C. Grant, OY

Wong, Sabrina, 19

Wu, Aiwen, 17, 1U, 1Y

Wu, Janet, 22

Wu, Xin, 1J

Xia, Annie, 1U, $1 Y$

Xie, Ting, 26

$X u$, Cheng Bai, 13

$X U$, Hong, $1 R$

Yaegashi, Hidetami, OK, $1 \mathrm{~N}$

Yamada, Koichi, 24

Yamada, Shintaro, 19

Yamamoto, Kimiko, 1A

Yamazaki, Akiyoshi, OU

Yang, Kou, $1 \mathrm{R}$

Yang, Michael, 26

Yoon, Byeri, 1D

Yoshida, Kohei, OU

Yoshida, Yuichi, OA

Yoshihara, Kosuke, OA

Yoshimura, Shota, $1 \mathrm{P}$

Yoshino, Takumi, OD

You, Gen, OW

Zhang, Baolin, 1G, $1 \mathrm{~J}$

Zhang, Keren, 19

Zhang, Lei, 19

Zhang, Libin, $1 \mathrm{~J}$

Zhang, Meiyue, 27

Zhang, Qi, 26

Zhang, Yuanyi, OQ, OR, 23

Zhang, Zhengping, $1 \mathrm{G}$

Zhu, Qingjun, OY 
Proc. of SPIE Vol. 10960 1096001-12 Downloaded From: https://www.spiedigitallibrary.org/conference-proceedings-of-spie on 26 Apr 2023
Terms of Use: https://www.spiedigitallibrary.org/terms-of-use 


\section{Conference Committee}

Symposium Chair

Will Conley, Cymer, an ASML Company (United States)

Symposium Co-chair

Kafai Lai, IBM Thomas J. Watson Research Center (United States)

Conference Chair

Roel Gronheid, KLA-Tencor//COS Belgium (Belgium)

Conference Co-chair

Daniel P. Sanders, IBM Research - Almaden (United States)

Conference Program Committee

Robert Allen, IBM Research - Almaden (United States)

Gilles R. Amblard, SAMSUNG Austin Semiconductor LLC (United States)

Ramakrishnan Ayothi, JSR Micro, Inc. (United States)

Ryan Callahan, FUJIFILM Electronic Materials U.S.A., Inc. (United States)

Joy Y. Cheng, Taiwan Semiconductor Manufacturing Company Ltd. (Taiwan)

Ralph R. Dammel, EMD Performance Materials Corporation (United States)

Anuja De Silva, IBM Corporation (United States)

Danilo De Simone, IMEC (Belgium)

Douglas J. Guerrero, Brewer Science, Inc. (United States)

Clifford L. Henderson, University of South Florida (United States)

Craig D. Higgins, GLOBALFOUNDRIES Inc. (United States)

Christoph K. Hohle, Fraunhofer-Institut für Photonische Mikrosysteme (Germany)

Scott W. Jessen, Texas Instruments Inc. (United States)

Yoshio Kawai, Shin-Etsu Chemical Company, Ltd. (Japan)

Qinghuang Lin, ASML US, Inc. (United States)

Nobuyuki N. Matsuzawa, Panasonic Corporation (Japan)

Steve S. Putna, Intel Corporation (United States)

Mark H. Somervell, Tokyo Electron America, Inc. (United States)

Jason K. Stowers, Inpria (United States)

James W. Thackeray, Dow Electronic Materials (United States) 
Raluca Tiron, CEA-LETI (France)

Rick Uchida, Tokyo Ohka Kogyo America, Inc. (United States)

Thomas I. Wallow, ASML Brion Technologies (United States)

\section{Session Chairs}

Opening Remarks and Award Announcements

Roel Gronheid, KLA-Tencor/ICOS Belgium (Belgium)

Daniel P. Sanders, IBM Research - Almaden (United States)

1 Keynote Session

Roel Gronheid, KLA-Tencor/ICOS Belgium (Belgium)

Daniel P. Sanders, IBM Research - Almaden (United States)

2 Inorganic Resists: Joint Session with Conferences 10960 and 10957

Robert L. Brainard, SUNY CNSE/SUNYIT (United States)

Jason K. Stowers, Inpria Corporation (United States)

3 Stochastics and Exposure Mechanisms: Joint Session with

Conferences 10960 and 10957

Marie Krysak, Intel Corporation (United States)

Thomas I. Wallow, ASML San Jose (United States)

4 EUV Resists

Danilo De Simone, IMEC (Belgium)

Anuja De Silva, IBM Corporation (United States)

5 Resist Fundamentals

Nobuyuki N. Matsuzawa, Panasonic Corporation (Japan)

Douglas J. Guerrero, Brewer Science, Inc. (Belgium)

6 Integration

Yoshio Kawai, Shin-Ełsu Chemical Co., Ltd. (Japan)

Ryan Callahan, FUJIFILM Electronic Materials U.S.A., Inc. (United States)

7 Monolayer Materials in Device Fabrication

Ralph R. Dammel, EMD Performance Materials Corporation (United States)

James W. Thackeray, Dow Electronic Materials (United States)

8 Directed Self-assembly I: Joint Session with Conferences 10960

and 10958

Raluca Tiron, CEA-LETI (France)

Ricardo Ruiz, HGST, Inc. (United States) 
9 Directed Self-assembly II: Defectivity

Mark H. Somervell, Tokyo Electron America, Inc. (United States)

Douglas J. Guerrero, Brewer Science, Inc. (United States)

10 Student Session

Scott W. Jessen, Texas Instruments Inc. (United States)

Robert D. Allen, IBM Research - Almaden (United States)

Poster Preview Speed Talks

Scott W. Jessen, Texas Instruments Inc. (United States)

Robert D. Allen, IBM Research - Almaden (United States)

11 Material Supplier

Christoph K. Hohle, Fraunhofer-Institut für Photonische Mikrosysteme (Germany)

Gilles R. Amblard, SAMSUNG Austin Semiconductor LLC

(United States)

12 Underlayers

Ryusuke Uchida, Tokyo Ohka Kogyo America, Inc. (United States)

Ramakrishnan Ayothi, JSR Micro, Inc. (United States)

Tribute to C. Grant Willson (retiring in 2019)

Qinghuang Lin, ASML US, Inc. (United States)

Roel Gronheid, KLA-Tencor/ICOS Belgium (Belgium) 
Proc. of SPIE Vol. 10960 1096001-16 Downloaded From: https://www.spiedigitallibrary.org/conference-proceedings-of-spie on 26 Apr 2023
Terms of Use: https://www.spiedigitallibrary.org/terms-of-use 